\title{
Science as a Social Good: Iramis Alonso-Porro Director, Juventud Técnica
}

\author{
Conner Gorry MA
}

Science journalism was little known in Cuba when Iramis Alonso wrote her thesis on the specialized field in 1990. That year, journalism degree from the University of Havana in hand, she set off to Cuba's eastern countryside to complete two years of social service reporting for local, regional and national print media. Living in the mountains of Holguín, a typical day for the cub reporter took her to caves, forests and fields for stories on the intersection of science, culture and the environment. Alonso credits this formative experience with igniting her passion for investigative and science journalism, setting her on a unique career path as a journalist and editor specializing in the sciences writ large: climate change, astronomy, mathematics and other hard sciences, engineering, information technologies and social sciences, among others.

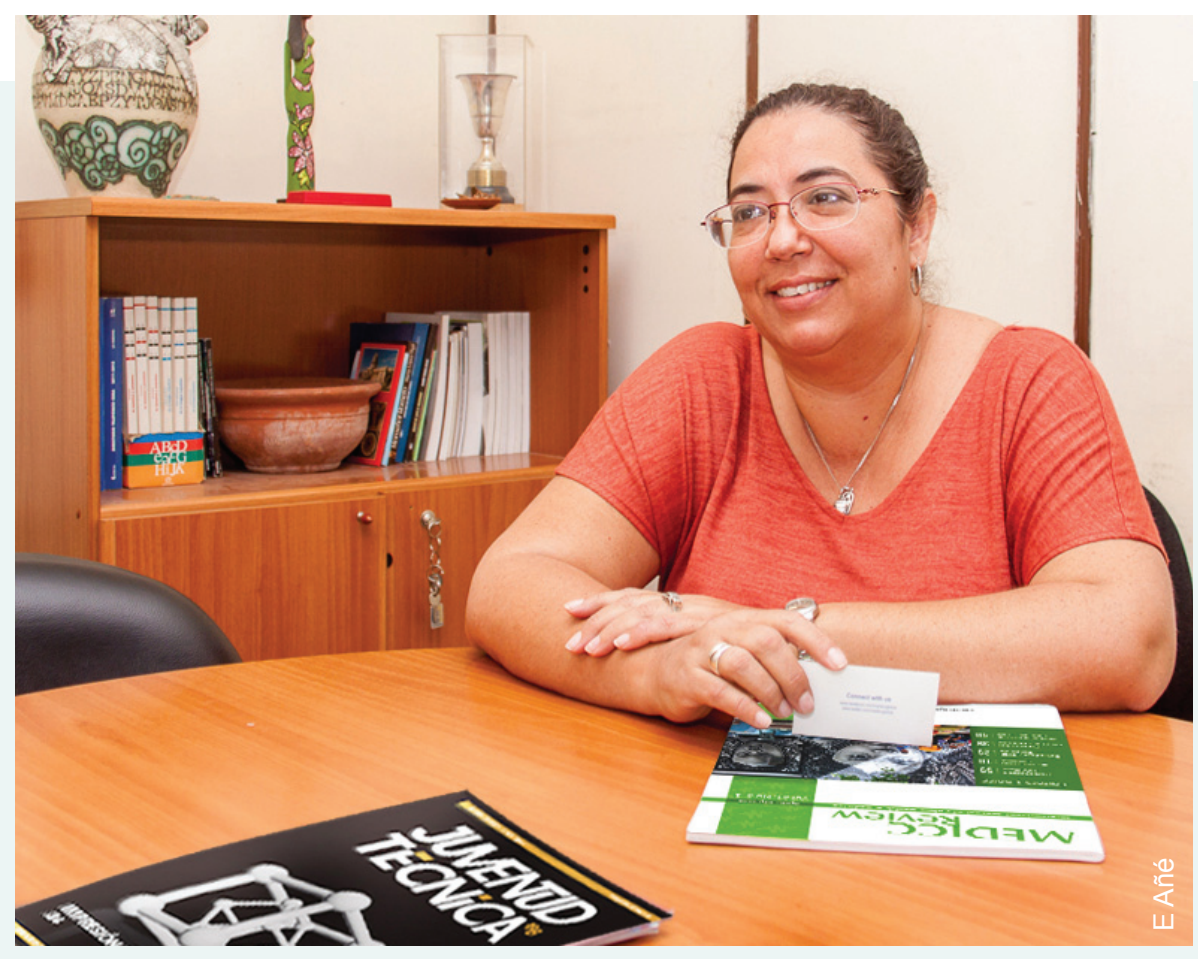

After finishing her social service, Alonso became a news analyst, parsing national and international print, radio and TV reports-this experience would prove indispensable in a future when misinformation can go viral. In 1994, Alonso began reporting on scientific issues for the national daily newspaper Juventud Rebelde where she spent five years broadening her knowledge and contacts and co-founding the paper's monthly scientific supplement, En Red, still published today. After eight years there, she jumped to Bohemia, Cuba's oldest and most respected magazine, where a culture of investigative journalism and 'pushing the envelope' prevails. She

MEDICC Review: Cubans revere Juventud Técnica (JT) as an entertaining and educational resource. But many MEDICC Review readers may never have heard of it. Can you talk a bit about the magazine's editorial mission?

Iramis Alonso: Juventud Técnica covers all fields of scienceastronomy, engineering, biotech and pharmaceutical development, the environment, mathematics and more-with the specific purpose of exploring the social applications of science. How can we get science out of the ivory tower and put it at the service of people to help improve their lives? That's our mission and vision. When considering editorial content, we focus on science as a social good and the magazine's contribution to moving that idea forward. So Juventud Técnica is not about science for the sake of science. Instead, we want to spark debate by covering polemical issues and digging deeper. So rather than report 'there's a novel vaccine and this is what it does' or 'new has worked with various international agencies, including the UN Development Programme (UNDP) and Oxfam, and was professor of science journalism at the University of Havana for six years (2011-2017). She is a regular lecturer on science journalism at the Jose Martí International Institute of Journalism in Havana. In 2007, Alonso became director of the national popular science magazine Juventud Técnica. In March, 2020, just two days after the first COVID-19 cases were confirmed in Cuba, she switched roles from journalist to interviewee. 
MEDICC Review: You helped spearhead science journalism and editing in Cuba as a specialized profession. Can you talk about how that happened and why it's important?

Iramis Alonso: Environmental reporting didn't become part of the University of Havana's journalism curriculum until 1992, following the Rio de Janeiro Earth Summit-so I didn't learn about it in the classroom, I learned about it in the field. During my social service, I was riding horses into the mountains to report on coffee yields or traveling to isolated coastal keys to write about beach erosion. I'll be honest: I miss field reporting.

When I became a science correspondent for Juventud Rebelde, I immediately became a part of the science and environment journalism section of the National Journalists Union. At the time, they had a really helpful system whereby once a month, we were invited on a site visit to different scientific institutions and research centers. We weren't expected to generate stories from these visits; they were meant for us to learn about the research happening and meet the scientists involved. One month we'd go to the Institute for Food Industry Research, the next to the Center for Applied Nuclear Technology Development and so on. This was an incredible learning experience and I wrote on just about every field of science while I was at the paper.

When I moved to Bohemia, where they employ specialist writers and editors for each section-culture, sports, science-I became convinced this is the ideal model. I firmly believe that scientific reporting and publishing requires a team of specialists, which is complex because science encompasses everything from computer technology and the environment to biotech and physics. We use the specialized journalism model at Juventud Técnica because readers need to know we've fact-checked and filtered the real from the bogus. It helps build and maintain credibility.

MEDICC Review: One of the issues tackled in your magazine and which you've written about falls under the social sciences umbrella-gender. How do you view the status of Cuban women in science today?

Iramis Alonso: Despite everything Cuba has achieved in gender parity and advancement of women in all senses, including science-the majority of scientists in Cuba are women!-we are still the minority in math and physics. There's a persistent sociocultural attitude that these fields are difficult, and more difficult endeavors are for men, while biology and chemistry are 'easier' and so somehow more 'appropriate' for women.

Of course we know that there is absolutely no evidence supporting this idea that biologically one gender is more intellectually suited or predisposed to one field over another. This bias is part of the machista construct and culture of our society. Stratifying social phenomena, instead of viewing them together as part of a diverse spectrum, also plays a part: who is to say that if I pursue a literature degree, that I don't also have the intellectual capacity to understand math or physics? This is a fairly generalized perception-that a person can't be drawn to or talented in several fields. When I was younger, I replicated this myth, saying "I'm happy not seeing another mathematical formula ever again." And I was good at math!

Breaking down this societal barrier and prejudice requires an integrated approach-in education, institutions and the media.
And it has to start in children's early developmental stages. We need more imagery and media coverage of women in diverse fields; more inclusive messaging starting in elementary school that girls can do anything boys can do; and to celebrate scientists returning from international congresses with press conferences at the airport like we do with athletes. Did you know that in 2017, the first Cuban woman took home 'perfect gold' from the Central American Math Olympics? Probably not, because there's not sufficient media coverage of her achievement-and she's a math superstar [Sofía Albizu-Campos Rodríguez, a 10th grader from Havana, won the gold medal with a perfect score in the 2017 competition-Eds.]

\section{MEDICC Review: Are there specific strategies Juventud} Técnica uses to promote women in science?

Iramis Alonso: Absolutely. We are especially pro-active when it comes to scientific citations. The thesis by a JT journalist examining the diversity of scientific citations in Cuban publications found that almost all references cited were by white men in their mid-50s or older. Given that most scientists in Cuba are women, we intuited that there must be women doing similar research in whatever field being cited. This led to a study we conducted on the h-index of Cuban scientists, which found that the two most-cited Cubans at that time were women-Dr María Guadalupe Guzmán and Dr Susana Vázquez, both of the Pedro Kourí Tropical Medicine Institute (IPK) in Havana. The Secretary of the Cuban Academy of Sciences, Dr Lilliam Álvarez, has made a point of trying to get more Cuban female scientists 'seen,' nominated for awards and recognized for their work, but the fact is, male scientists are more visible.

So citing references, we consult our colleagues at the Cuban Academy of Sciences and scientific institutions to see if there is an appropriate reference by a woman or person of color we might substitute for the older white male citation. We consciously look for these alternatives. It goes without saying that first and foremost, the reference must be valid and meet the scientific criteria-no personal characteristics can supersede that.

In our interview section, we consciously strike a balance by interviewing as many women as men, and people of different skin color and ages: our goal is to represent the reality of Cuban science and that includes all types of people. By publishing interviews with younger scientists we also hope to stimulate the new generation to pursue scientific careers.

We are conscious about the language we use in our articles as well. Instead of saying 'science in the service of man,' we say 'science in the service of the human race,' which is more accurate anyway. And because Spanish uses grammatical gender [with gendered nouns and defaulting to the masculine when pluralized-Eds.] we look for gender-neutral alternatives.

MEDICC Review: The title of your publication includes 'youth.' Is it difficult attracting young people to scientific fields in Cuba?

Iramis Alonso: This is extraordinarily complex given Cuba's context. On the one hand, our education system graduates highcaliber professionals-despite resource scarcity and brain drain of professors to other countries and the private sector. On the 


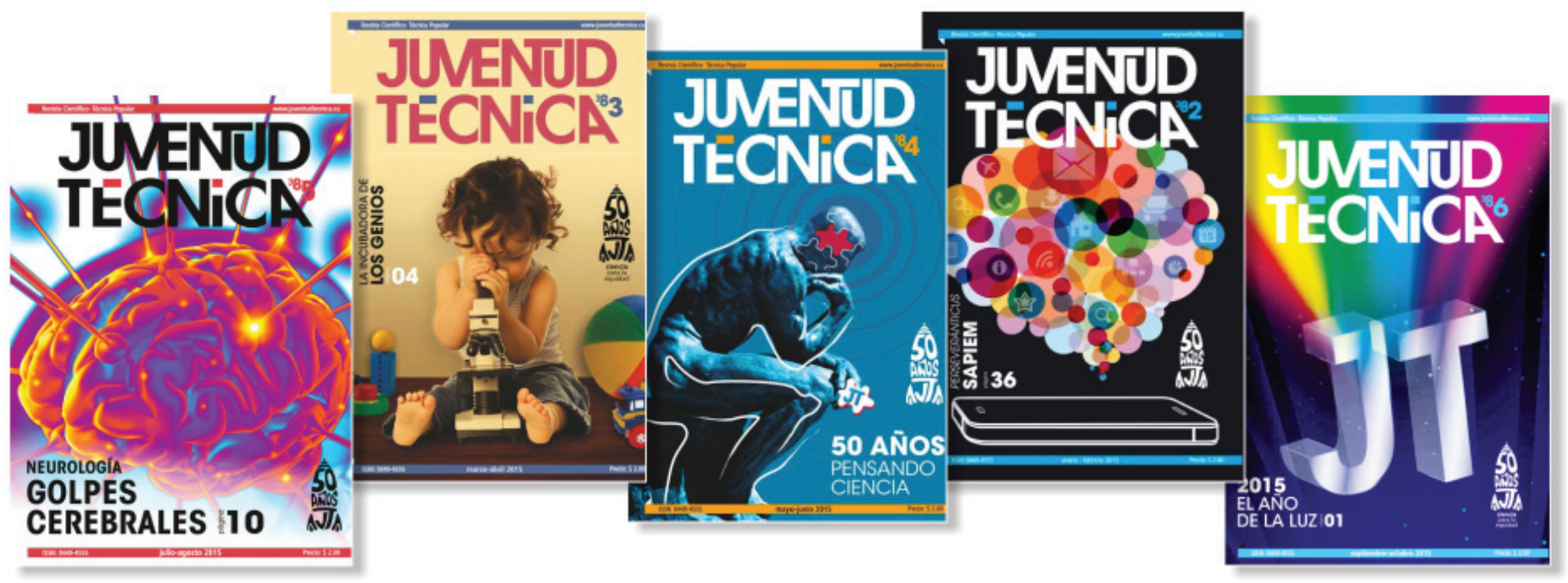

other hand, we don't have the economy to match, so it's difficult to attract young people into science. In short, Cuba has a firstworld professional capacity within a developing-world economy, compounded by the US embargo of course.

So how do we reconcile the younger generation's social and professional commitment with their individual goals given the country's tenuous economic circumstances? This is no small challenge since we need their energy and creativity to develop sustainably.

The network of scientific and technological campuses cropping up across the country is a very interesting advance in this regard. These act as incubators for innovation, linking businesses, research institutes and universities to spark scientific and technological solutions to societal problems, while generating revenue for the country and the professionals forging those solutions. Supported by financial mechanisms and incentives, this model presents the possibility for more efficient implementation of applied research, greater student participation, and more passion in general for science and technology as young people see their ideas put into practice. The earning potential of this intersectoral approach is also much faster than traditional scientific research and development.

\section{MEDICC Review: Undoubtedly, human resources are one of Cuba's strengths....}

Iramis Alonso: Cuba doesn't have significant natural resource deposits or export market possibilities. And while I don't particularly like the term 'human resources'—we're talking about people!-it is shorthand for where we excel: a highly educated and trained professional and technical workforce. But it's not only about providing good jobs for this workforce, because one of the challenges we're facing is related to the perceived value of science. This is more intangible but no less important. It boils down to what value does science have for Cuba and for humanity? Doing more to impassion our society about science is needed. Going to the theater or movies, listening to music-these are popular pastimes. Imagine if looking at the stars and identifying constellations like I used to do with my grandfather or going to the natural sciences museum or aquarium became as popular...All the things we're talking about need to spring from a strong scientific culture.
MEDICC Review: Speaking of passions, what are you most passionate about in your work?

Iramis Alonso: I feel like my mission in life-aside from raising my kids, one of whom is a physicist, so I'm doing my part for science there as well!-is to mentor young journalists. I want to nurture their interest in scientific reporting, editing and publishing so the field continues to thrive and grow. JT is the only publication of its kind and we function as a forge for the next generation of scientific journalists and editors. This type of training and experience is important since we'll need to pass the torch to them at some point.

My other passion is to continue learning for my personal and professional edification. l've had no shortage of opportunities in this regard since l'm not a digital native and JT is very much in the thick of new technologies, with new multimedia platforms and initiatives.

\section{MEDICC Review: What are some of these new initiatives?}

Iramis Alonso: Despite challenges we've faced with the digital version of $J T$-streaming audio and video was problematic given our limited bandwidth-we are getting ready to launch JT 4.0. This is a multimedia platform with audiovisual components that incorporates a more user friendly and attractive interface without sacrificing scientific rigor. And this is both the driving force and biggest hurdle with new media and technologies for us as a publication: how do we design a social media and online strategy that pulls in young readers but maintains quality reporting? Too much of what's published online these days defaults to sensationalism just to get as many clicks as possible. And while we understand that remaining relevant to younger generations, who are digital natives, means having shorter, snappier headlines, using eye-catching graphics and being more agile in our reporting and response to readers, we still need to maintain the evidencebased and scientific standards for which we're known.

Doing this successfully requires us to adapt and learn new skills; a print publication is a different animal-slower, more in-depthbut we've developed a strategy and are about to hire a digital community manager to help us maximize the potential of the medium. This is especially important since we began our digital fact-checking initiative \#VerificaJT. 
MEDICC Review: This project was mentioned in MEDICC Review's April 2020 issue in relation to coronavirus misinformation and disinformation. Can you explain what this is and why it's necessary?

Iramis Alonso: We launched \#VerificaJT to combat false news in science, technology, health and the environment. It's readergenerated and designed to fact check rumors and disinformation in these fields. Readers tag us with \#VerificaJT about items or reports they think be false or need accuracy checked and our journalists begin to research the news in question to determine whether it's true or not.

Specialized science publishing The glut of information helps filter out the real and valid news from the partially true or outright fabricated today is astounding; there's more information out there than human beings can process and then there's the question of how to process it, to filter it. So this, too, is a function of specialized science editing and publishing: it helps filter out the real and valid news from the partially true or outright fabricated. This is incredibly basic for me as a journalist-our job is to root out and verify facts, the credibility of sources, always going to the original source. When was it published? Where? What type of publication is it? Is their editorial process rigorous? These are the questions we ask when we get a reader request via \#VerificaJT.

This is where our social media strategy comes in. Since we follow so many scientists and specialists on Twitter, for example, they can be consulted speedily to help verify news in their field. Twitter has proven an extraordinary tool for us in this regard, but also because you can tag who you want to see your content-if we're writing about gender violence, we tag the Cuban Women's Federation or Oxfam, both of which have campaigns against gender violence. Facebook is more like a crowded apartment building where all the neighbors are giving their opinion, so its less useful, but in Cuba you have to be on Facebook since its where the overwhelming majority of Cubans are online and provides the most visibility. We have strategies for both, plus Instagram and Telegram, too.

\section{MEDICC Review: Can you give some examples of items} readers have sent in to \#VerificaJT?

Iramis Alonso: First, let me say that there is so much fabricated news flying around on the internet, we could dedicate our entire work day to just this. So we prioritize the fields I mentioned and serious inquiries only. We aren't interested in those queries sent by people determined to provoke or stir the pot.

So while we've received a lot of requests, we've only addressed a handful. One was a rumor that was making the rounds about contaminated Arcor food products [Arcor is an Argentina-based food company with 40 industrial plants in Latin America; their products are sold in Cuba-Eds]. We began researching reports and after consulting a similar factchecking project in Argentina called Chequeado, learned that this was a two-year old rumor that started in that country and was completely false. We also addressed a rumor in Cuba about bananas being injected with HIV-contaminated blood. Also false. We had a reader request about a tornado that touched down in Mayabeque Province, not far from Havana. This was easy to verify as true, since journalists from the regional newspaper in Mayabeque reported on it.

More recently, we debunked a rumor about the first coronavirus cases in Cuba. On February 27, 2020, news began circulating on social media that there were three tourists with coronavirus hospitalized at the IPK. Since we have a large network of contacts at that hospital and research institute, it was an easy and quick process to verify that this was false news [the first three conf rmed cases of coronavirus in Cuba were diagnosed on March 11, 2020, Italian tourists who had arrived March 9-Eds]. Having contacts such as these and at the Cuban Academy of Sciences and other research institutes is a tremendous resource for quick and accurate verification.

MEDICC Review: Cuba's National COVID Prevention and Control Plan was adopted over a month before the first cases were detected. How might the pandemic and the control measures affect your work?

Iramis Alonso: One of the hundreds of measures put into place here relates to telecommuting, something we've been doing at $J T$ for some time. Actually, I came in today just to talk to you; usually, we're only in the office once a week for our editorial planning meeting. We meet each week to design our work plan, divvy up editorial assignments and responsibilities and then work online from home. We've had to adapt this to our context and connectivity challenges, with low-data usage workarounds to send documents back and forth for example, but it's effective. This work-from-home model has advantages, especially since some of our team members live far away. While telecommuting for us predates COVID, it will become important for many different sectors as transportation and physical contact becomes more restricted.

I foresee a lot of requests via \#VerificaJT-social media is dominated by COVID-content content right now, not all factbased, as we know. We've also been generating a constant stream of infographics and launched a COVID-19 dashboard, Covid19CubaData, with all the latest data, available online (https://covid19cubadata.github.io/) and as a mobile app (https:// www.apklis.cu/application/ club.postdata.covid19cuba).

What I can tell you is our work is about to become a lot more intense! -1/

Editors' Note: In follow-up emails through early July, 2020, Iramis Alonso indicated that work at JT had indeed intensified and showed no signs of slowing, but noted the positive progress made in controlling COVID-19 in Cuba. As of this writing, the entire country has entered Phase 2 of the re-opening plan, except Havana which continues in Phase 1; the capital cannot pass to Phase 2 until the 5 established epidemiological criteria are met.

Published July 31, 2020 https:doi.org/10.37757/MR2020.V22.N3.4 